\title{
Some Inequalities for the $q$-Analogue of the Classical Riemann Zeta Functions and the $q$-Polygamma Functions
}

\section{Banyat Sroysang \\ Department of Mathematics and Statistics, Faculty of Science and Technology, Thammasat University, Pathumthani 12121, Thailand}

Correspondence should be addressed to Banyat Sroysang; banyat@mathstat.sci.tu.ac.th

Received 30 August 2013; Accepted 12 November 2013; Published 22 January 2014

Academic Editors: A. Barbagallo and Y.-M. Chu

Copyright (C) 2014 Banyat Sroysang. This is an open access article distributed under the Creative Commons Attribution License, which permits unrestricted use, distribution, and reproduction in any medium, provided the original work is properly cited.

We present the generalizations on some inequalities for the $q$-analogue of the classical Riemann zeta functions and the $q$-polygamma functions.

\section{Introduction and Preliminaries}

Let $c \in \mathbb{C}$ and $q \in(0,1)$. The $q$-shifted factorial $[1-5]$ is defined by

$$
(c ; q)_{\infty}=\prod_{k=0}^{\infty}\left(1-c q^{k}\right) .
$$

The $q$-Jackson integral [6] from 0 to $c$ is defined by

$$
\int_{0}^{c} f(x) d_{q} x=(1-q) c \sum_{n=0}^{\infty} f\left(c q^{n}\right) q^{n} .
$$

The q-gamma function [6] is defined by

$$
\Gamma_{q}(x)=\frac{(q ; q)_{\infty}}{\left(q^{x} ; q\right)_{\infty}}(1-q)^{1-x}, \quad x \neq 0,-1,-2, \ldots
$$

The q-digamma [7-9] function is defined by

$$
\psi_{q}(x)=\frac{\Gamma_{q}^{\prime}(x)}{\Gamma_{q}(x)}
$$

and may be represented [10] as

$$
\psi_{q}(x)=-\log (1-q)+\frac{\log q}{1-q} \int_{0}^{q} \frac{t^{x-1}}{1-t} d_{q} t,
$$

where $x>0$.
The $q$-polygamma function [7] is defined by

$$
\psi_{q, n}(x)=\psi_{q}^{(n)}(x)
$$

and may be represented as

$$
\psi_{q, n}(x)=\frac{\log q}{1-q} \int_{0}^{q} \frac{(\log u)^{n} u^{x-1}}{1-u} d_{q} u,
$$

where $x>0$ and $n \in \mathbb{N}$.

For any $x>0$, we denote

$$
\begin{gathered}
\alpha(x)=\frac{\log x}{\log q}-E\left(\frac{\log x}{\log q}\right), \\
{[x]_{q}=\frac{1-q^{x}}{1-q},} \\
\{x\}_{q}=\frac{[x]_{q}}{q^{x+\alpha\left([x]_{q}\right)}},
\end{gathered}
$$

where $E(\log x / \log q)$ is the integer part of $\log x / \log q$.

The $q$-zeta function [11] is defined by

$$
\zeta_{q}(s)=\sum_{n=1}^{\infty} \frac{1}{\{n\}_{q}^{s}},
$$

where $\operatorname{Re}(s)>1$. Moreover, in [11], the $q$-analogue of the classical Riemann Zeta function is

$$
\zeta_{q}(s)=\frac{1}{\widetilde{\Gamma}_{q}(s)} \int_{0}^{\infty} t^{s-1} Z_{q}(t) d_{q} t,
$$


where $\operatorname{Re}(s)>1$ and for any $t>0$,

$$
\begin{gathered}
Z_{q}(t)=\sum_{n=1}^{\infty} \frac{1}{\left((q-1)\{n\}_{q}^{t} ; q\right)_{\infty}}, \\
\widetilde{\Gamma}_{q}(s) \\
=\frac{\left(1+(1-q)^{-1}\right)\left((q-1) q^{t} ; q\right)_{\infty}\left((q-1)^{-1} q^{1-t} ; q\right)_{\infty}}{\left((1-q)^{-s}(q-1) ; q\right)_{\infty}\left((q-1)^{-1} ; q\right)_{\infty}} \Gamma_{q}(s) .
\end{gathered}
$$

In 2009, Brahim [12] proved the results as follows:

$$
\psi_{q, m}(x) \psi_{q, n}(x) \geq \psi_{q,(m+n) / 2}^{2}(x),
$$

where $x>0$ and $m, n,(m+n) / 2 \in \mathbb{N}$. Consider

$$
[s+1]_{q} \frac{\zeta_{q}(s)}{\zeta_{q}(s+1)} \geq q[s]_{q} \frac{\zeta_{q}(s+1)}{\zeta_{q}(s+2)},
$$

where $s>1$.

In 2010, Krasniqi et al. [13] gave an inequality as follows:

$$
\frac{\widetilde{\Gamma}_{q}(x / a+y / b)}{\widetilde{\Gamma}_{q}^{1 / a}(x) \widetilde{\Gamma}_{q}^{1 / b}(y)} \leq \frac{\zeta_{q}^{1 / a}(x) \zeta_{q}^{1 / b}(y)}{\zeta_{q}(x / a+y / b)}
$$

where $x, y, a>1$ and $x / a+y / b>1=1 / a+1 / b$.

In 2012, Sulaiman [10] gave the inequalities as follows:

$$
\psi_{q, m / a+n / b}\left(\frac{x}{a}+\frac{y}{b}\right) \leq \psi_{q, m}^{1 / a}(x) \psi_{q, n}^{1 / b}(y),
$$

where $x, y>0, a>1,1 / a+1 / b=1$, and $m, n, x / a+y / b \in \mathbb{N}$.

$$
\left(\psi_{q, m}(x)+\psi_{q, n}(y)\right)^{1 / p} \leq \psi_{q, m}^{1 / p}(x)+\psi_{q, n}^{1 / p}(y)
$$

where $x, y>0, p \geq 1$, and $m, n \in \mathbb{N}$.

In this paper, we present the generalizations on the above inequalities.

\section{Results}

Theorem 1. Let $n_{1}, n_{2}, \ldots, n_{k} \in \mathbb{N}, x_{1}, x_{2}, \ldots, x_{k}>0$, and $p_{1}, p_{2}, \ldots, p_{k}>1$ be such that $\sum_{i=1}^{k} 1 / p_{i}=1$ and $M=$ $\sum_{i=1}^{k} n_{i} / p_{i} \in \mathbb{N}$. Then

$$
\psi_{q, M}\left(\sum_{i=1}^{k} \frac{x_{i}}{p_{i}}\right) \leq \prod_{i=1}^{k} \psi_{q, n_{i}}^{1 / p_{i}}\left(x_{i}\right) .
$$

Proof. By the generalized Hölder inequality,

$$
\begin{aligned}
\psi_{q, M}\left(\sum_{i=1}^{k} \frac{x_{i}}{p_{i}}\right) & =\frac{\log q}{1-q} \int_{0}^{q} \frac{(\log u)^{M} u^{\sum_{i=1}^{k} x_{i} / p_{i}-1}}{1-u} d_{q} u \\
& =\frac{\log q}{1-q} \int_{0}^{q} \frac{(\log u)^{\sum_{i=1}^{k} n_{i} / p_{i}} u^{\sum_{i=1}^{k}\left(x_{i}-1\right) / p_{i}}}{(1-u)^{\sum_{i=1}^{k}\left(1 / p_{i}\right)}} d_{q} u \\
& =\frac{\log q}{1-q} \int_{0}^{q} \prod_{i=1}^{k}\left(\frac{(\log u)^{n_{i} / p_{i}} u^{\left(x_{i}-1\right) / p_{i}}}{(1-u)^{1 / p_{i}}}\right) d_{q} u \\
& \leq \frac{\log q}{1-q} \prod_{i=1}^{k}\left(\int_{0}^{q} \frac{(\log u)^{n_{i}} u^{x_{i}-1}}{1-u} d_{q} u\right)^{1 / p_{i}} \\
& =\prod_{i=1}^{k} \psi_{q, n_{i}}^{1 / p_{i}}\left(x_{i}\right) .
\end{aligned}
$$

One can easily check that if we put $k=2$ in Theorem 1 then we get the following.

Corollary 2 (see [10]). Let $n_{1}, n_{2} \in \mathbb{N}, x_{1}, x_{2}>0$, and $p_{1}, p_{2}>$ 1 be such that $1 / p_{1}+1 / p_{2}=1$ and $n_{1} / p_{1}+n_{2} / p_{2} \in \mathbb{N}$. Then

$$
\psi_{q, n_{1} / p_{1}+n_{2} / p_{2} s}\left(\frac{x_{1}}{p_{1}}+\frac{x_{2}}{p_{2}}\right) \leq \psi_{q, n_{1}}^{1 / p_{1}}\left(x_{1}\right) \psi_{q, n_{2}}^{1 / p_{2}}\left(x_{2}\right) \text {. }
$$

It is easy to notice that if we put $k=p_{1}=p_{2}=2$ and $x_{1}=x_{2}=x$ in Theorem 1 then we get the following.

Corollary 3 (see [12]). Let $n_{1}, n_{2} \in \mathbb{N}$ and $x>0$ be such that $\left(n_{1}+n_{2}\right) / 2 \in \mathbb{N}$. Then

$$
\psi_{q,\left(n_{1}+n_{2}\right) / 2}^{2}(x) \leq \psi_{q, n_{1}}(x) \psi_{q, n_{2}}(x)
$$

Theorem 4. Let $n_{1}, n_{2}, \ldots, n_{k} \in \mathbb{N}, x_{1}, x_{2}, \ldots, x_{k}>0$, and $p \geq 1$. Then

$$
\left(\sum_{i=1}^{k} \psi_{q, n_{i}}\left(x_{i}\right)\right)^{1 / p} \leq \sum_{i=1}^{k} \psi_{q, n_{i}}^{1 / p}\left(x_{i}\right)
$$

Proof. First, we note that

$$
\sum_{i=1}^{k} a_{i}^{p} \leq\left(\sum_{i=1}^{k} a_{i}\right)^{p}
$$

for all $a_{1}, a_{2}, \ldots, a_{k}>0$. Then

$$
\begin{aligned}
& \left(\sum_{i=1}^{k} \psi_{q, n_{i}}\left(x_{i}\right)\right)^{1 / p} \\
& \quad=\left(\sum_{i=1}^{k}\left(\frac{\log q}{1-q} \int_{0}^{q} \frac{(\log u)^{n_{i}} u^{x_{i}-1}}{1-u} d_{q} u\right)\right)^{1 / p}
\end{aligned}
$$




$$
\begin{aligned}
& =\left(\frac{\log q}{1-q}\right)^{1 / p}\left(\int_{0}^{q} \sum_{i=1}^{k}\left(\frac{(\log u)^{n_{i} / p} u^{\left(x_{i}-1\right) / p}}{(1-u)^{1 / p}}\right)^{p} d_{q} u\right)^{1 / p} \\
& \leq\left(\frac{\log q}{1-q}\right)^{1 / p}\left(\int_{0}^{q}\left(\sum_{i=1}^{k} \frac{(\log u)^{n_{i} / p} u^{\left(x_{i}-1\right) / p}}{(1-u)^{1 / p}}\right)^{p} d_{q} u\right)^{1 / p} .
\end{aligned}
$$

By Minkowski’s inequality,

$$
\begin{aligned}
& \left(\sum_{i=1}^{k} \psi_{q, n_{i}}\left(x_{i}\right)\right)^{1 / p} \\
& \quad \leq\left(\frac{\log q}{1-q}\right)^{1 / p} \sum_{i=1}^{k}\left(\int_{0}^{q}\left(\frac{(\log u)^{n_{i} / p} u^{\left(x_{i}-1\right) / p}}{(1-u)^{1 / p}}\right)^{p} d_{q} u\right)^{1 / p} \\
& \quad=\sum_{i=1}^{k}\left(\frac{\log q}{1-q} \int_{0}^{q}\left(\frac{(\log u)^{n_{i}} u^{x_{i}-1}}{1-u}\right)^{p} d_{q} u\right)^{1 / p} \\
& \quad=\sum_{i=1}^{k} \psi_{q, n_{i}}^{1 / p}\left(x_{i}\right) .
\end{aligned}
$$

One can easily check that if we put $k=2$ in Theorem 4 then we get the following.

Corollary 5 (see [10]). Let $n_{1}, n_{2} \in \mathbb{N}, x_{1}, x_{2}>0$, and $p \geq 1$. Then

$$
\left(\psi_{q, n_{1}}\left(x_{1}\right)+\psi_{q, n_{2}}\left(x_{2}\right)\right)^{1 / p} \leq \psi_{q, n_{1}}^{1 / p}\left(x_{1}\right)+\psi_{q, n_{2}}^{1 / p}\left(x_{2}\right) .
$$

Theorem 6. Let $x_{1}, x_{2}, \ldots, x_{k}, p_{1}, p_{2}, \ldots, p_{k}>1$ be such that $\sum_{i=1}^{k} x_{i} / p_{i}>1=\sum_{i=1}^{k} 1 / p_{i}$. Then

$$
\zeta_{q}\left(\sum_{i=1}^{k} \frac{x_{i}}{p_{i}}\right) \leq \frac{\prod_{i=1}^{k} \widetilde{\Gamma}_{q}^{1 / p_{i}}\left(x_{i}\right)}{\widetilde{\Gamma}_{q}\left(\sum_{i=1}^{k} x_{i} / p_{i}\right)} \prod_{i=1}^{k} \zeta_{q}^{1 / p_{i}}\left(x_{i}\right) .
$$

Proof. By the generalized Hölder inequality,

$$
\begin{aligned}
& \zeta_{q}\left(\sum_{i=1}^{k} \frac{x_{i}}{p_{i}}\right) \\
& =\frac{1}{\widetilde{\Gamma}_{q}\left(\sum_{i=1}^{k} x_{i} / p_{i}\right)} \int_{0}^{\infty} t^{\sum_{i=1}^{k} x_{i} / p_{i}-1} Z_{q}(t) d_{q} t \\
& =\frac{1}{\widetilde{\Gamma}_{q}\left(\sum_{i=1}^{k} x_{i} / p_{i}\right)} \int_{0}^{\infty} t^{\sum_{i=1}^{k}\left(x_{i}-1\right) / p_{i}} Z_{q}^{\sum_{i=1}^{k} 1 / p_{i}}(t) d_{q} t \\
& =\frac{1}{\widetilde{\Gamma}_{q}\left(\sum_{i=1}^{k} x_{i} / p_{i}\right)} \int_{0}^{\infty} \prod_{i=1}^{k}\left(t^{x_{i}-1} Z_{q}(t)\right)^{1 / p_{i}} d_{q} t \\
& \quad \leq \frac{1}{\widetilde{\Gamma}_{q}\left(\sum_{i=1}^{k} x_{i} / p_{i}\right)} \prod_{i=1}^{k}\left(\int_{0}^{\infty} t^{x_{i}-1} Z_{q}(t) d_{q} t\right)^{1 / p_{i}}
\end{aligned}
$$

$$
\begin{aligned}
= & \frac{1}{\widetilde{\Gamma}_{q}\left(\sum_{i=1}^{k} x_{i} / p_{i}\right)} \\
& \times \prod_{i=1}^{k}\left(\widetilde{\Gamma}_{q}^{1 / p_{i}}\left(x_{i}\right)\left(\frac{1}{\widetilde{\Gamma}_{q}\left(x_{i}\right)} \int_{0}^{\infty} t^{x_{i}-1} Z_{q}(t) d_{q} t\right)^{1 / p_{i}}\right) \\
= & \frac{\prod_{i=1}^{k} \widetilde{\Gamma}_{q}^{1 / p_{i}}\left(x_{i}\right)}{\widetilde{\Gamma}_{q}\left(\sum_{i=1}^{k} x_{i} / p_{i}\right)} \prod_{i=1}^{k} \zeta_{q}^{1 / p_{i}}\left(x_{i}\right) .
\end{aligned}
$$

It is easy to notice that if we put $k=2$ in Theorem 6 then we get the following.

Corollary 7 (see [13]). Let $x_{1}, x_{2}, p_{1}, p_{2}>1$ be such that $x_{1} / p_{1}+x_{2} / p_{2}>1=1 / p_{1}+1 / p_{2}$. Then

$$
\frac{\widetilde{\Gamma}_{q}\left(x_{1} / p_{1}+x_{2} / p_{2}\right)}{\widetilde{\Gamma}_{q}^{1 / p_{1}}\left(x_{1}\right) \widetilde{\Gamma}_{q}^{1 / p_{2}}\left(x_{2}\right)} \leq \frac{\zeta_{q}^{1 / p_{1}}\left(x_{1}\right) \zeta_{q}^{1 / p_{2}}\left(x_{2}\right)}{\zeta_{q}\left(x_{1} / p_{1}+x_{2} / p_{2}\right)} .
$$

\section{Conflict of Interests}

The author declares that there is no conflict of interests regarding the publication of this paper.

\section{Acknowledgments}

The author would like to thank the referees for their useful comments and suggestions.

\section{References}

[1] G. Gasper and M. Rahman, Basic Hypergeometric Series, Encyclopedia of Mathematics and Its Applications, Cambridge University Press, Cambridge, UK, 2nd edition, 2004.

[2] V. G. Kac and P. Cheung, Quantum Calculus, Universitext, Springer, New York, NY, USA, 2002.

[3] P. M. Rajkovic and S. D. Marinkonvic, "Fractional integrals and derivative in q-calculus," Applicable Analysis and Discrete Mathematics, vol. 1, pp. 311-323, 2007.

[4] B. Sroysang, "An Inequality for the q-polygamma function," Advanced Studies in Theoretical Physics, vol. 7, no. 14, pp. 693696, 2013.

[5] B. Sroysang, "A study on a new fractional integral inequality in quantum calculus," Advanced Studies in Theoretical Physics, vol. 7, no. 14, pp. 689-692, 2013.

[6] F. H. Jackson, “On a q-definite integrals," The Quarterly Journal of Pure and Applied Mathematics, vol. 41, no. 2, pp. 193-203, 1910.

[7] K. Brahim and S. Taf, "Some fractional integral inequalities in quantum calculus," Journal of Fractional Calculus and Applications, vol. 4, no. 2, pp. 245-250, 2013.

[8] A. De Sole and V. G. Kac, "On integral representations of qgamma and q-beta functions," Atti della Accademia Nazionale dei Lincei, Classe di Scienze Fisiche, Matematiche e Naturali, Rendiconti Lincei Matematica E Applicazioni, vol. 16, no. 1, pp. 11-29, 2005. 
[9] A. Fitouhi and K. Brahim, "Tauberian theorems in quantum calculus," Journal of Nonlinear Mathematical Physics, vol. 14, no. 3, pp. 324-340, 2007.

[10] W. T. Sulaiman, "Turan type inequalities for some special functions," Australian Journal of Mathematical Analysis and Applications, vol. 9, no. 1, article 1, 7 pages, 2012.

[11] A. Fitouhi, N. Bettaibi, and K. Brahim, "The mellin transform in quantum calculus," Constructive Approximation, vol. 23, no. 3, pp. 305-323, 2006.

[12] K. Brahim, "Turán-type inequalities for some $q$-special functions," Journal of Inequalities in Pure and Applied Mathematics, vol. 10, no. 2, article 50, 5 pages, 2009.

[13] V. Krasniqi, T. Mansour, and A. S. Shabani, "Some inequalities for $q$-polygamma function and $\zeta q$-Riemann zeta functions," Annales Mathematicae et Informaticae, vol. 37, no. 1, pp. 95-100, 2010. 


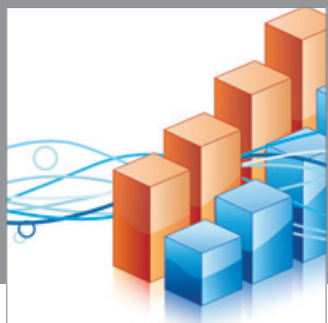

Advances in

Operations Research

mansans

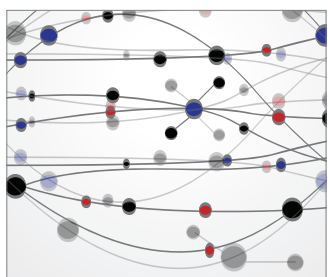

The Scientific World Journal
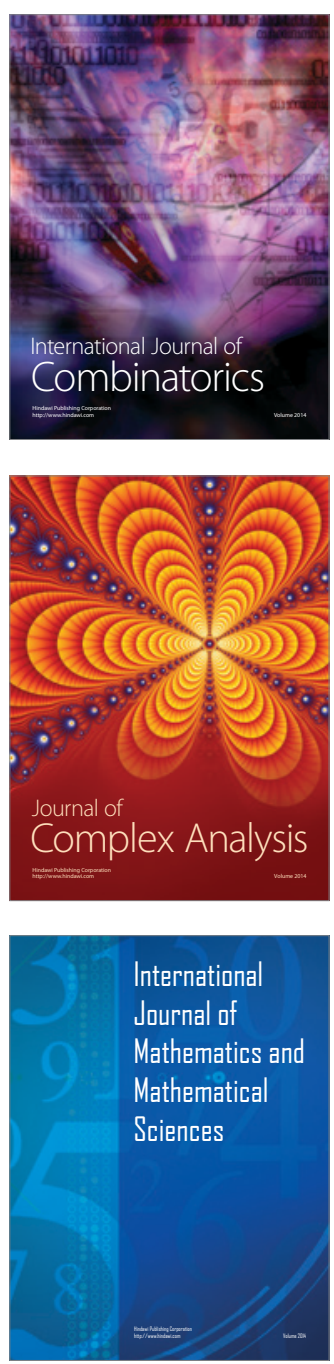
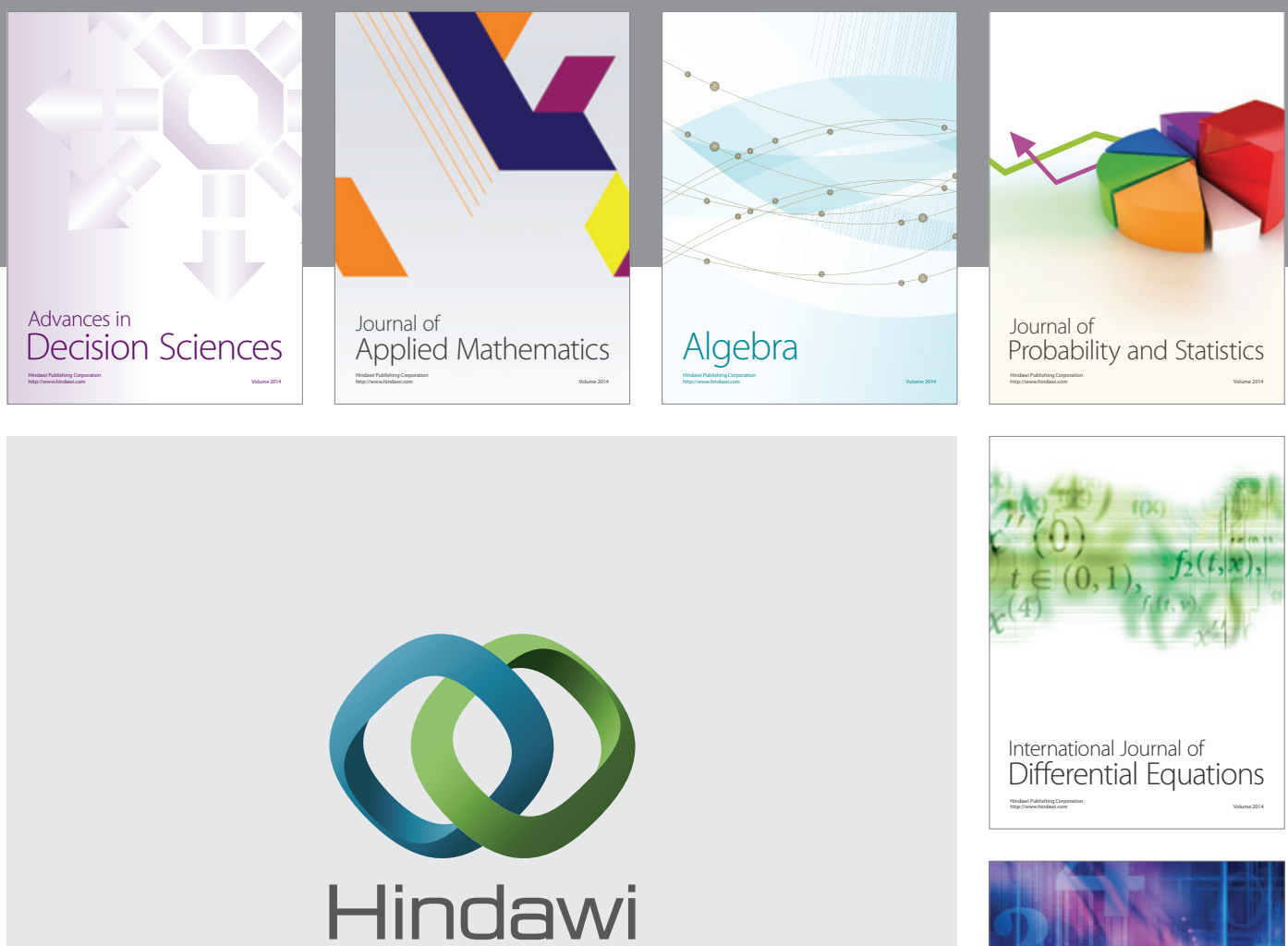

Submit your manuscripts at http://www.hindawi.com
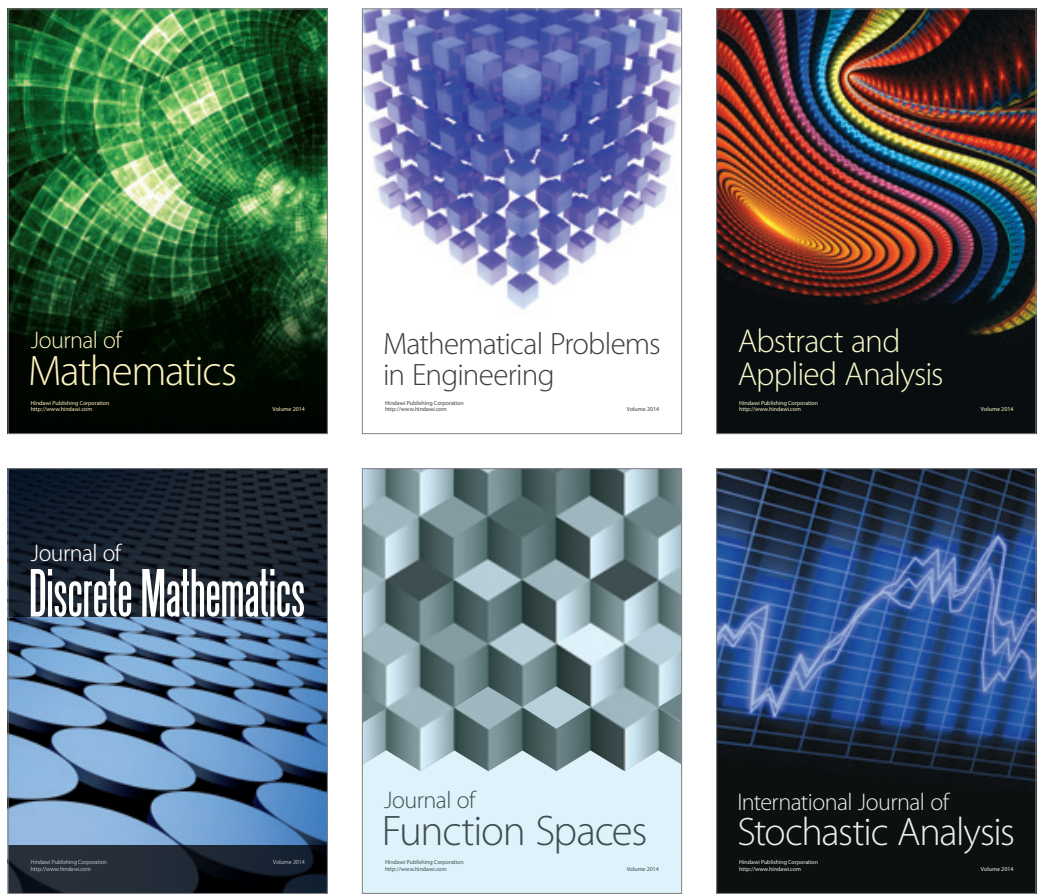

Journal of

Function Spaces

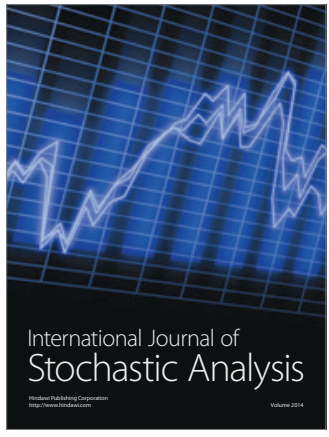

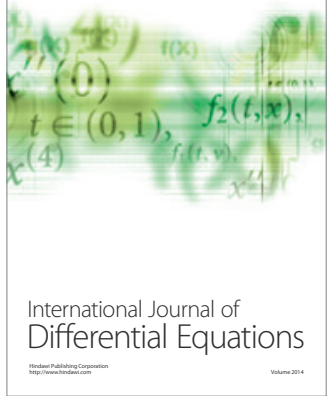
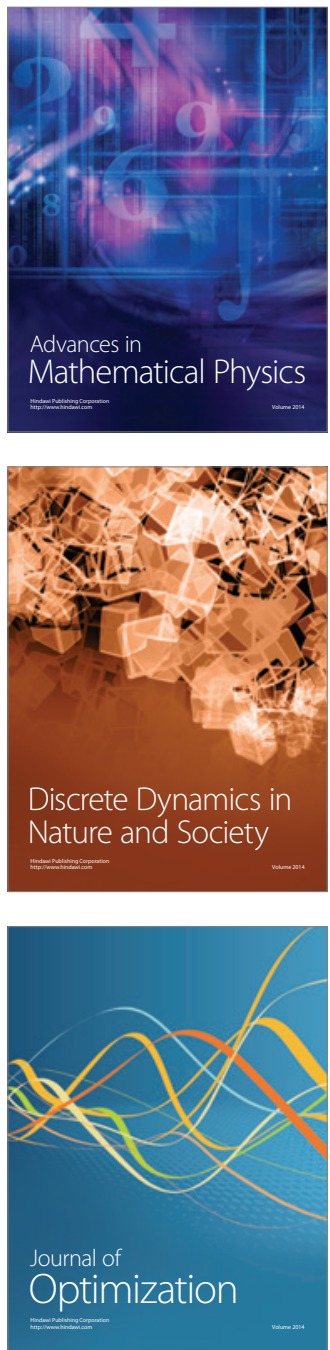